\title{
Short-term results of Proximal humeral locking plate fixation in displaced proximal humerus fractures.
}

\author{
Mohd Yaseen Rather ${ }^{1}$, Mohd Shafi Bhat ${ }^{2}$, Mohd Rafiq Bhat ${ }^{3}$, Riyaz Ahmad \\ Dar $^{4}$, Mubashir Beigh ${ }^{5}$, Mohd Ramzan Mir ${ }^{6}$. \\ $1 \& 3$ : Postgraduate Scholars Orthopaedics . \\ 2 : Postgraduate Scholar and Correspondence Author. \\ 4 \& 5 : Senior residents Orthopaedics. \\ 6: Professor and Head Department of Orthopaedics. \\ Hospital for Bone and Joint Surgery Barzulla Srinagar Kashmir (India). \\ (An associated hospital of Govt Medical College Srinagar.)
}

\begin{abstract}
A case series of 25 cases ( 21 male and 4 female ) of displaced proximal humerus fractures operated at Hospital for Bone and Joint Surgery Barzulla Srinagar Kashmir (India) using the proximal humeral locking plate. The study was conducted from Nov 2010 to Oct 2012.The average age of patients was 45.5 years (range 24-92). Neer's classification was used for classifying the fractures. There were 4 patients with 4-part fracture, 12 with 3-part fracture and 9 patients with 2-part fractures. All the cases were operated within 3 weeks of the injury. The mean follow-up time was 13.4 months. Average time to union was 15.6 weeks. Final assessment was done using Constant Murley Shoulder Score. Excellent or good results were obtained in 20, fair results in 2 and poor results in 2 patients.
\end{abstract}

Keywords: Proximal humeral fractures, locking plate fixation, internal fixation.

\section{Introduction}

The proximal humeral fractures constitute 4-5\% of all fractures and $45 \%$ of humeral fractures. Most of these fractures occur in woman over the age of 50 with the exception of isolated greater tuberosity fractures which occur with increased frequency in young individuals. More than $85 \%$ of these fractures are minimally displaced or undisplaced and are effectively treated symptomatically with immobilization followed by early motion. ${ }^{1,2}$ Various techniques are available to surgeon fixing proximal humeral fracture and they include minimally invasive techniques like use of krischner wires, tension band, cerclage wires, T-plates, intramedullary devices,prosthetic replacements , the plan tan humerus fixator plate, the polaris nail etc $^{3,4,5,6,7}$. However,complications have been reported using these techniques including cutouts and backout of screws and plates, avascular necrosis, nonunion, malunion, nail migration, rotator cuff impingement and impingement syndrome ${ }^{4,7}$. The rate of these complications have approached as high as $100 \%$ for elderly people ${ }^{6,7}$.

The Proximal humeral locking plate is a new addition to vast number of techniques for fixation of proximal humeral fractures ${ }^{8}$.The screws in head are locked into plate and cannot back and toggle, which is particularly advantageous in osteoporotic bone ${ }^{9}$. The screws alternatively converge and diverge thus improving fixation strength, particularly in osteoporotic bone of elderly. The plate being low profile minimizes the risk of impingement. ${ }^{10}$

\section{Materials And Method}

This prospective study is a series of 25 patients who were operated from November 2010 to October 2012 for proximal humerus fractures using proximal humeral locking plate. The study was conducted at Hospital for Bone and Joint Surgery Barzulla Srinagar Kashmir. Displaced proximal humeral fractures in patients with age > 18 years were included in the study. Undisplaced fractures, open fractures and 4-part fracture-dislocations were excluded from the study. The results were evaulated using Constant Murley Shoulder score $^{11}$.

Plain radiographs (antero-posterior, axillary and lateral views ) were obtained preoperatively to access the fracture pattern. The operation ${ }^{12,13,14}$ was performed under GA in supine position with head rotated to the contralateral side and well supported in head rest. Standard deltopectoral approach was used in all the cases. The fracture fragments were restroted to their best possible anatomic position with minimal soft tissue 
dissection. Preliminary reduction was held with use of Krishner wires. Reduction was assessed under image intensifier .Definitive fixation with Proximal humeral locking plate was done with plate positioned at least $5 \mathrm{~mm}$ distal to the upper end of greater tuberosity to prevent subacromial impingement and atleast $2 \mathrm{~mm}$ posterior to bicipital groove thus sparing the tendon of long head of biceps. Plate was positioned to bone in desired position first with use of K-wires and then definitive fixation achieved with use of multidirectional locking screws. After plate application range of motion was checked in all directions under image intensifier to look for any impingement and screw penetration into the joint. Wound was closed over suction drain and shoulder immobilizer applied.

Passive ROM exercises were started within 24-48 hours. ${ }^{12,13,14}$ Shoulder immobilizer was used for a period of 4 weeks, with immobilizer removed at the time of ROM exercises. The active exercises were started after one month .

\section{Results}

There were 21 males and 4 females in our study. The average age of our patients was $45.5 y e a r s$ (range 24-92 ). The mechanism of injury was fall in 13 patients, road traffic accidents in 11 patients and direct hit to shoulder in 1 patient. In our study we had 4 four part fractures, 12 three part fractures and 9 two part fractures. We operated on right side in 15 patients and on left side in 10 patients. $44 \%$ of patients in our study were operated in first week, another $44 \%$ in $2^{\text {nd }}$ week, 3 patients reported after two weeks of injury, nevertheless we operated all our patients before 3 weeks. The mean follow-up time was 13.4 months. Average time for union in our study was 15.6 weeks with no case of nonunion. We assessed our patients using Constant Murley Shoulder Score $^{11}$. We achieved good or excellent results in 20 patients, fair in 2 patients and poor in 3 patients. 5 patients in our study achieved ROM less than satisfactory, while 20 patients achieved excellent or good range of motion. Out of 25 patients, 21 had no pain, 2 patients had occasional pain usually seasonal and 2 patients had pain related to activity.

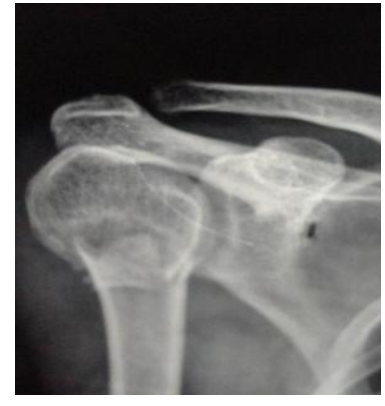

Fig 1 . X-ray AP view shoulder with 2-part fracture of proximal humerus.

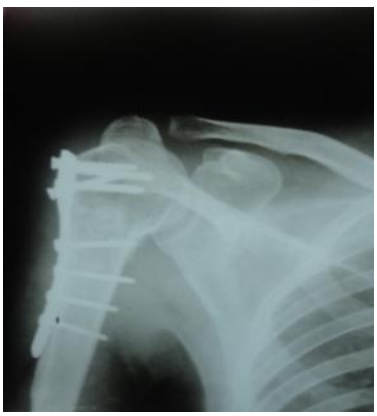

Fig 2. Post-op X-ray showing the proximal humerus locking plate fixation.

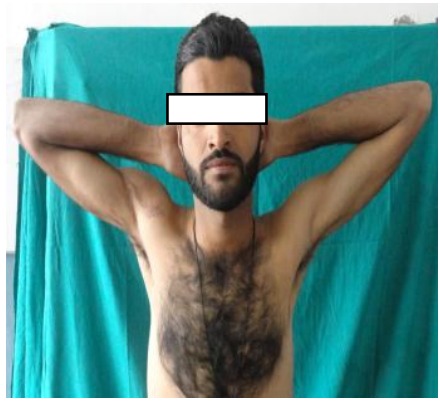

Fig 3. ROM shoulder at final follow-up.

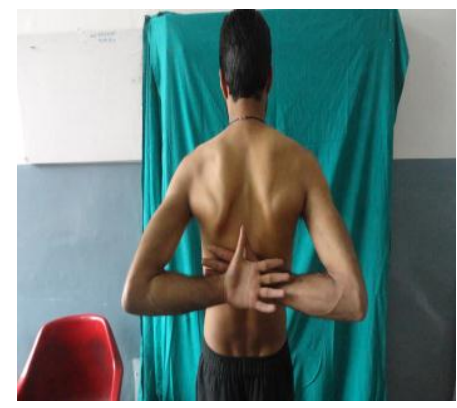

Fig 4. ROM shoulder at final follow-up.

\section{Discussion}

Fracture of proximal humerus is still the unsolved fracture in many ways. Much controversy and 
confusion still exists and no treatment protocol or algorithm has been proved to be universally effective. There is disagreement with regard to reliability of classification systems. The indications for surgical management continue to be modified.

S.K Moda ${ }^{15}$ in 1990 reported open reduction and internal fixation in 25 severely displaced fractures and fracture dislocations of proximal humeral fractures with $\mathrm{T}$ plate and semi-tubular plate and reported good or excellent results in $84 \%$ patients ${ }^{17}$. Wijgman ${ }^{16}$ in 2002 reported good and excellent results in $87 \%$ of patients treated with T-buttress plate or cerclage wire in three and four part fractures. Hintermann et $\mathrm{al}^{17}$ reported favorable results with blade plate.

Over a period of time new techniques have been introduced for treatment of proximal humeral fractures. Rajasekhar et $\mathrm{al}^{4}$ worked on polaris nail and reported $80 \%$ satisfactory to excellent results. They operated on 23 two part fractures out of total 27 patients. Proximal humeral locking plate for fixation of proximal humerus fractures is a new addition to the vast number of techniques used for fixation of these fractures. It is a locking plate with advantages like strong fixation, high union rates and decreased incidence of hardware failure ${ }^{12,13,14}$.In a retrospective study Bjorkenheim et $\mathrm{al}^{9}$ reviewed 72 patients treated with PHILOS Plate.36 patients had good or excellent functional outcome. In a series of 20 patients Atanosis Koukakis et al. showed favourable early results with surgical management of proximal humeral fractures using PHILOS plate.

We assessed our patients using Constant Murley Shoulder Score ${ }^{11}$. Excellent or good results were obtained in $80 \%$ of patients. The benefit of Proximal humeral locking plate over other techniques available for fixation of proximal humeral fractures is its superior fixation even in osteoporotic bone of elderly with low incidence of screw back out and cutout as observed by many authors ${ }^{12,13,14}$. The plate being low profile decreases the incidence of impingement ${ }^{12,13}$. The fixation being superior helps in starting early ROM exercises and hence superior results in the long term. The plate is situated posterior to biceps tendon avoiding damage to anterior humeral circumflex artery, thus decreasing the risk of avascular necrosis.

We had complications in the form of superficial infection in one patient which healed with intravenous antibiotics. There was no case of deep infection in our study.

\section{Conclusion}

We can conclude from our observations that proximal humeral locking plate fixation is a successful procedure. Strong fixation provided by Proximal humeral locking plate assists in starting early postoperative ROM exercises.

\section{Bibliography}

[1.] Court-Brown CM, Garg A, McQueen MM. The epidemiology of proximal humeral fractures. Acta Orthop Scand 2001;72(4):365371.

[2.] Chu SP, Kelsey JL, Keegan TH, Sternfeld B et al: Risk factors for proximal humeral fracture.Am J epidemiol 2004;160(4):360367.

[3.] Park MC, Murthi AM, Roth NS, Blaine TA, Levine WN, Biglaini LU. Two-part and three-part fractures of proximal humerus treated with suture fixation. J Orthop Trauma.2003;17(5):319-325.

[4.] Rajasekhar C, Ray PS, Bhamra MS. Fixation of proximal humeral fractures with the Polarus nail. J Shoulder Elbow Surg.2001;10(1):7-10.

[5.] Robinson CM, Page RS, Hill RM,s anders DL, Court-Brown CM, Waverfield AE. Primary hemiarthroplasty for treatment of proximal humeral fractures. J Bone Joint Surg.2003;85A:1215-1223.

[6.] Sadowski C, Riand N, Stern R, Hoffmeyer P. Fixation of fractures of the proximal humerus with the plan tan humerus fixation plate: Early experience with a new implant. J Shoulder Elbow Surg.2003;12:148-151.

[7.] Sehr JR, Szabo RM. Semitubular blade plate for fixation in the proximal humerus. J Orthop Trauma.1998;2: 327-332.

[8.] Seidel H. Humeral locking nail. A preliminary report.Orthopaedics.1989;12:219-226.

[9.] Bjorkenheim JM, Pajarinen J, Savolainen V. Internal fixation of proximal humeral fractures with a locking compression plate:a retrospective evaluation of 72 patients followed for a minimum of 1 year. Acta Orthop Scand 2004:75(6):741-5.

[10.] Koukakis A, Apostoulou MD, Taneja T, Korres DS, Amini A. Fixation of proximal humeral fractures using the PHILOS Plate: early experience. Clin orthop Relat Res 2006;(442):115-20.

[11.] Constant C R, Murley AH. A clinical method of functional assessment of the shoulder. Clin Orthop Relat Res 1987; 214:160-164.

[12.] Sameer Aggarwal, Kamal Bali , Mandeep S Dhillon, Vishal Kumar, Aditya K Mootha .Displaced proximal humeral fractures:an Indian experience with locking plates .J Orthop Surg Research 2010;5:60.

[13.] Bulent KILIC,Mustafa UYSAL ,Bekir murat CINAR ,Gurkan OZKOC, Huseyin DEMIRORS , Secran AKPINAR Early results of treatment of proximal humeral fractures with the PHILOS locking plate. Acta Orthop Traumatol Turc 2008 ;42(3):149-153.

[14.] Mehmet Fatih KORKMAZ, Neslihan AKSU, Abdullah GOGUS, Mursel DEBRE,Ayhan Nedim KARA,Zekeriya Ugur ISIKLAR .The results of internal fixation of proximal humeral fractureswith the PHILOS locking plate.Acta Orthop Traumatol Turc 2008;42 (2) : $97-105$

[15.] SK Moda,N S Chadha, S S Sangwan, D K Khurana et al.Open reduction and internal fixation of proximal humeral fractures and 
fracture dislocations. J Bone Joint Surg Br 1990;72-B:1050-2.

[16.] Wijgman AJ,Roolker W,Patt TW,Raaymakers EL,Marti RK. Open reduction and internal fixation of three and four part fractures of proximal part of the humerus.J Bone Joint Surg 2002;84A(11):1919-1925.

[17.] Hintermann B, Troullier HH, Schafer D. Rigid internal fixation of fractures of the proximal humerus in older patient. J Bone Joint Surg 2000;82(8):1107-1112. 